\title{
Compelling reasons why viruses are relevant for the origin of cells
}

\author{
Eugene V. Koonin, Tatiana G. Senkevich and Valerian V. Dolja
}

We discuss below the interesting recent article by Moreira and López-García (Ten reasons to exclude viruses from the tree of life. Nature Rev. Microbiol. 7, 306-311 $(2009))^{1}$. Thanks to the discovery of the giant Mimivirus ${ }^{2}$ and advances of viral metagenomics that establish viruses as a quantitatively dominant component of the biosphere ${ }^{3}$, the evolution of viruses has become a topic of lively discussion ${ }^{2,4-8}$. Several scenarios were proposed according to which viruses antedated cells and contributed to their origin ${ }^{4,5,7}$. The Opinion article by Moreira and LópezGarcía extends this discussion ${ }^{1}$ by making three fundamental points: viruses are not alive, contrary to claims that they are ${ }^{2,5}$; viruses do not belong in the tree of life (TOL); and viruses are not ancient and are irrelevant for the reconstruction of cellular evolution.

The first point is more metaphysical than scientific. Viruses are objects of biology, and many key discoveries of molecular biology were made using virus models. We are content to leave the quest for the 'best' definition of life to philosophers. However, some of the arguments of Moreira and López-García, in particular, that viruses "evolved by cells" and lack "structural historical continuity", are worth attention. We think that these statements are false. Not only do viruses demonstrate evolutionary continuity, but large gene sets evolve congruently over hundreds of millions of years, for example, in the nucleocytoplasmic large DNA viruses (NCLDVs) of eukaryotes ${ }^{9}$. Alive or 'undead', viruses are distinct, evolving genetic entities that collectively constitute the virus world (virosphere), a crucial part of the biome of our planet.

The second point is ironic because evolutionary genomics shows that the TOL as an 'organismal tree' does not exist ${ }^{10}$. Genes have distinct evolutionary trajectories owing to duplication, lineage-specific gene loss and horizontal gene transfer (HGT): there are few if any fully consistent gene trees. Viruses do not have universal genes, whereas cellular life forms possess a universal gene core. However, that core is tiny ${ }^{11}$, so that attempts to construct a TOL from universal genes ${ }^{12}$ yield, at best, a "tree of one percent" (REF. 13). Whether or not a TOL can be salvaged as some central trend in the thicket of the phylogenetic forest remains an open question, but evolutionary trees of viral genes legitimately belong in that forest.

The third point is most important and is the reason we feel compelled to respond. Moreira and López-García recognize that dissimilar viruses infecting widely different hosts share related genes. However, they dismiss these viral hallmark genes (VHGs) as evidence of the existence of an ancient, pre-cellular virus world ${ }^{4}$ on the basis of three arguments: convergence, interviral HGT and horizontal transfer of viruses between divergent hosts. The argument of convergence cannot be taken seriously. Even for capsid proteins of icosahedral viruses, convergence is not a viable option considering the conservation of the topology of the jelly roll fold ${ }^{6}$. For the other VHGs, there is highly significant sequence similarity between homologous genes from widely different viruses ${ }^{4}$, rendering convergence a moot issue.

Interviral HGT is a demonstrable phenomenon'. However, HGT is not so rampant as to obliterate the signal of vertical evolution, even among tailed bacteriophages that are notoriously prone to genetic mobility ${ }^{14}$. Moreover, HGT between viruses with different replication strategies, such as large DNA viruses and positive-strand RNA viruses, has not been detected; nevertheless, these viruses share VHGs. Thus, the most parsimonious explanation for the existence of VHGs that are shared by an enormous range of viruses but possess only distant homologues among cellular genes is that they are relics of pre-cellular evolution ${ }^{4}$.

Moreira and Lopez-Garcia argue that the wide spread of many viral groups among diverse host taxa (such as NCLDV $^{9}$ or picorna-like viruses ${ }^{8}$ ) is not evidence of the antiquity of these viruses owing to horizontal virus transfer (HVT). However, HVT has its limits: to our knowledge, no viruses cross the boundary between prokaryotes and eukaryotes. In sharp contrast, several VHGs are shared by prokaryotic and eukaryotic viruses ${ }^{4}$. Even the potentially more plausible movement of viruses between unicellular and multicellular eukaryotes has not been documented, and is an unlikely explanation for the broad host range of the major groups of viruses ${ }^{4,8}$.

A pre-cellular, 'virus-like' stage of evolution appears to be, effectively, a logical inevitability. It is unimaginable that life started with large and complex genomes that were similar to those of archaea and bacteria, especially within the framework of the RNA world hypothesis, because large RNA genomes could not exist owing to RNA fragility. Thus, a pre-cellular stage of evolution must have involved genetic elements of virus-like size and complexity: the selfish replicons ${ }^{4,7}$. Importantly, unlike cellular life forms, viruses exploit different genome replication strategies, including those that were indispensable in the RNA world and during the transition to a DNA world, namely, RNA-dependent synthesis of RNA and DNA. The corresponding enzymes, the RNA-dependent RNA polymerases and reverse transcriptases, are bona fide VHGs that are shared by vast virus groups ${ }^{4}$. Conceivably, the diverse viral genomic strategies are relics of the primordial stage of evolution from which one strategy was selected by cells and the rest remained within the virus world.

Moreira and López-García emphasize the origin of all viral genes that are related to translation and energy conversion through gene transfer from hosts, and suggest that these observations imply the evolution of viral genomes from cellular genetic material ${ }^{1}$. Massive acquisition of host genes by large DNA viruses is undeniable ${ }^{9}$, but the contribution of this route is highly non-uniform among viruses. Small viral genomes have few if any genes of cellular origin and consist, mostly, of $\mathrm{VHGs}^{4}$. The most consistent explanation for the presence of VHGs in both the most complex and the simplest viruses is that they are relics of pre-cellular evolution. In any system of co-evolving genetic elements, segregation into parasites and hosts is inevitable because replication is an inherently selfish process ${ }^{15}$. Under the primordial virus world concept, those genetic elements that retained genes supporting translation, energy transformation and other metabolic processes gave rise to cells, whereas those that lost or never had those genes became viruses. 


\section{CORRESPONDENCE}

Cellular origin is one of the hardest problems in biology. The virus world seems to provide a much-needed window into the key, early stages of this process. We think that this window should not be shut out of simplistic considerations that, mostly, boil down to the notion that extant viruses are obligate intracellular parasites and are therefore irrelevant to the origin of cells.

Eugene V. Koonin is at the National Center for Biotechnology Information, National Library of Medicine National Institutes of Health, Bethesda, Maryland 20894, USA.

Tatiana G. Senkevich is at the Laboratory of Viral Diseases, National Institutes of Allergy and Infectious Diseases, National Institutes of Health, Bethesda, Maryland 20894, USA.
Valerian V. Dolja is at the Department of Botany and Plant Pathology and Center for Genome Research and Biocomputing, Oregon State University, Corvallis, Oregon 97331, USA

Correspondence to E.V.K e-mail:koonin@ncbi.nlm.nih.gov

1. Moreira, D. \& López-García, P. Ten reasons to exclude viruses from the tree of life. Nature Rev. Microbiol. 7 , 306-311 (2009).

2. Raoult, D. $\&$ Forterre, P. Redefining viruses: lessons from Mimivirus. Nature Rev. Microbiol. 6, 315-319 (2008).

3. Edwards, R. A. \& Rohwer, F. Viral metagenomics. Nature Rev. Microbiol. 3, 504-510 (2005).

4 Koonin, E. V., Senkevich, T. G. \& Dolja, V. V. The ancient virus world and evolution of cells. Biol. Direct 1, 29 (2006).

5. Claverie, J. M. Viruses take center stage in cellular evolution. Genome Biol. 7, 110 (2006).

6. Krupovic, M. \& Bamford, D. H. Virus evolution: how far does the double $\beta$-barrel viral lineage extend? Nature Rev. Microbiol. 6, 941-948 (2008).

7. Forterre, $P$. The origin of viruses and their possible roles in major evolutionary transitions. Virus Res. 117, 5-16 (2006).
8. Koonin, E. V., Wolf, Y. I., Nagasaki, K. \& Dolja, V. V. The Big Bang of picorna-like virus evolution antedates the radiation of eukaryotic supergroups. Nature Rev. Microbiol. 6, 925-939 (2008).

9 lyer, L. M., Balaji, S., Koonin, E. V. \& Aravind, L. Evolutionary genomics of nucleo-cytoplasmic large DNA viruses. Virus Res. 117, 156-184 (2006).

10. Doolittle, W. F. \& Bapteste, E. Pattern pluralism and the tree of life hypothesis. Proc. Natl Acad. Sci. USA 104, 2043-2049 (2007).

11. Charlebois, R. L. \& Doolittle, W. F. Computing prokaryotic gene ubiquity: rescuing the core from extinction. Genome Res. 14, 2469-2477 (2004)

12. Ciccarelli, F. D. et al. Toward automatic reconstruction of a highly resolved tree of life. Science 311, 1283-1287 (2006).

13. Dagan, T. \& Martin, W. The tree of one percent. Genome Biol. 7, 118 (2006)

14. Glazko, G., Makarenkov, V., Liu, J. \& Mushegian, A Evolutionary history of bacteriophages with double-stranded DNA genomes. Biol. Direct 2, 36 (2007).

15. Eigen, M. Selforganization of matter and the evolution of biological macromolecules. Naturwissenschaften 58, 465-523 (1971). 